\title{
SUPPORT THEOREMS FOR RADON TRANSFORMS ON HIGHER RANK SYMMETRIC SPACES
}

\author{
FULTON GONZALEZ AND ERIC TODD QUINTO
}

(Communicated by J. Marshall Ash)

\begin{abstract}
We prove support theorems for Radon transforms with real-analytic measures on horocycles in higher rank symmetric spaces. The microlocal analysis is more difficult than for rank one, but we prove a generalization of Helgason's support theorem and a theorem that is new even in the classical case.
\end{abstract}

\section{INTRODUCTION}

In this paper, we prove support theorems for Radon transforms over horocycles on noncompact symmetric spaces $G / K$ of arbitrary rank. The measures on the horocycles are assumed to be real-analytic and nowhere zero but are otherwise arbitrary. Theorem 4.1 generalizes Helgason's support theorem for the classical horocycle transform on $G / K$. His theorem is equivalent to the Paley-Wiener theorem for the Fourier transform on $G / K$ and is used to prove the global solvability of invariant differential operators on $G / K[10]$.

Our research is based on the pioneering work of Helgason (e.g., $[9,10])$ on group-invariant Radon transforms and Guillemin and Sternberg $[4,5,6]$ on Radon transforms as Fourier integral operators. Other authors have used Fourier integral techniques to understand Radon transforms (e.g., $[1,3,19$, 21]). This article is a natural successor to [17] in which the rank one case is studied. The rank one case is easier because all possible wavefront directions are detected by Radon transform data (see Theorem 3.1).

Helgason [10] proved the support theorem for the classical horocycle transform using global methods; i.e., the expansion of smooth functions on $G / K$ and $G / M N$ via the $L^{2}$ Fourier series on $K / M$. Our methods here are purely local, employing the theory of real-analytic Fourier integral operators (Theorem 3.1 ) to deduce analytic smoothness of a distribution $f$ from support restrictions on $R_{\mu} f$. Then a theorem of Hörmander, Kawai, and Kashiwara [14, 15] about analytic singularities and support is used to deduce support restrictions on $f$ from analytic smoothness of $f$. These local techniques allow us to prove a support theorem, Theorem 4.2, that is new even in the classical case.

Received by the editors October 5, 1992 and, in revised form, March 2, 1993.

1991 Mathematics Subject Classification. Primary 44A12; Secondary 58G15, 43 A85.

Key words and phrases. Radon transform on horocycles, symmetric space, support theorems, microlocal analysis.

The second author was partially supported by NSF grants MCS 8901203 and MCS 9123862 . 
The Radon transform, $R_{\mu}$, is defined using the group double fibration in $\S 2$. The microlocal analysis is given in $\S 3$ and the support theorems, Theorems 4.1 and 4.2, are proven in $\S 4$. Generalizations of Theorem 4.1 are given in $\S 5$.

\section{THE POINT-HOROCYCLE DOUBLE FIBRATION}

Let $G$ be a real semisimple noncompact Lie group. Let $G=K A N$ be an Iwasawa decomposition and let $M$ be the centralizer of $A$ in $K$. The pointhorocycle double fibration is given by:

$$
\begin{gathered}
Z=G / M \stackrel{p_{Y}}{\rightarrow} \quad Y=G / M N \\
\downarrow p_{X} \\
X=G / K
\end{gathered}
$$

where $p_{X}$ and $p_{Y}$ are the natural maps [12]. For each $\xi \in Y$, there corresponds a unique closed horocycle in $X, \hat{\xi}=p_{X} p_{Y}^{-1}(\{\xi\})$. For $x \in G / K$, define $\check{x}=$ $p_{Y} p_{X}^{-1}(\{x\})$. Because the maps in (2.1) are fibrations, all $\hat{\xi}$ are diffeomorphic (to $N$ ), and all $\check{x}$ are diffeomorphic (to $K / M$ ) [7,12]. The set $Z=G / M$ can be imbedded in $X \times Y$ as $Z=\{(x, \xi) \in X \times Y \mid x \in \hat{\xi}\}$, and the maps $p_{X}$ and $p_{Y}$ are projections under this identification. Also, $\check{x}$ can be viewed as the set of all horocycles through $x$.

Let $\mu(x, \xi)$ be a nowhere zero real-analytic function for $(x, \xi) \in Z$. The Radon transform of a function $f \in C_{c}(X)$ is defined to be

$$
R_{\mu} f(\xi)=\int_{x \in \hat{\xi}} f(x) \mu(x, \xi) d m_{\xi}(x)
$$

where $d m_{\xi}$ is the canonical measure on $\hat{\xi}$ given from the Haar measure on $G$ [7]. This is the integral of $f$ over the horocycle $\hat{\xi} \subset X$ with respect to the realanalytic measure $\mu d m_{\xi}$. The dual Radon transform of a function $g \in C(Y)$ is defined in a similar way,

$$
R_{\mu}^{*} g(x)=\int_{\xi \in \dot{x}} g(\xi) \mu(x, \xi) d m_{x}(\xi)
$$

where $d m_{x}$ is the canonical measure on $\check{x}$ given from the Haar measure on $G$ [7]. The transform $R_{\mu}^{*}: C^{\infty}(Y) \rightarrow C^{\infty}(X)$ is continuous for similar reasons to those given in [12, Chapter 1, Proposition 3.8] for $\mu=1$. Therefore, $R_{\mu}$ can be extended to domain $\mathscr{E}^{\prime}(G / K)$ by duality. In particular, for $f \in \mathscr{E}^{\prime}(X)$, we define $R_{\mu} f \in \mathscr{C}^{\prime}(Y)$ by the relation $\left\langle R_{\mu} f, g\right\rangle=\left\langle f, R_{\mu}^{*} g\right\rangle$ for $g \in C^{\infty}(Y)$.

Nonstandard measures are interesting because canonical measures do not occur in general, and our proofs, involving microlocal analysis, are valid for fairly general measures. Using nonstandard measures can help focus on properties intrinsic to the Radon transform rather than on specific symmetry relations that are valid only for special cases.

\section{THE MICROLOCAL ANALYSIS OF $R_{\mu}$}

Because $G / K$ is a real-analytic manifold, the analytic wavefront set, $\mathrm{WF}_{\mathrm{A}}(f)$, of a distribution $f \in \mathscr{E}^{\prime}(X)$ can be defined using local coordinates and the definition in $\mathbb{R}^{\mathrm{n}}[20]$; $\mathrm{WF}_{\mathbf{A}}(f)$ is a subset of the cotangent space $T^{*} X$.

The key to the support theorems is the way that $R_{\mu}$ detects singularities, Theorem 3.1. This is described in terms of Lie algebras. Let $\mathfrak{g}, \mathfrak{k}, \mathfrak{a}, \mathfrak{m}$, and 
$\mathfrak{n}$ be the Lie algebras of $G, K, A, M$, and $N$, respectively. Recall that the element $S \in \mathfrak{a} \backslash 0$ is regular if $\alpha(S) \neq 0$ for all restricted roots $\alpha$ (see [11, p. 292]). Let $\mathfrak{a}^{\prime}$ denote the set of regular elements in $\mathfrak{a}$.

Let $\mathfrak{g}=\mathfrak{k} \oplus \mathfrak{p}$ be the Cartan decomposition of $\mathfrak{g}$, and let $B$ denote the Killing form on $\mathfrak{g}$. Recall that $\left.B\right|_{\mathfrak{k}}$ is negative definite; $\left.B\right|_{\mathfrak{p}}$ is positive definite; and that $\mathfrak{g}=\mathfrak{k} \oplus \mathfrak{p}$ is an orthogonal decomposition with respect to $B$ [11, Proposition III 7.4]. Identify $\mathfrak{g}^{*}$ with $\mathfrak{g}$ using $B$. If $\mathfrak{h} \subset \mathfrak{g}$, then define

$$
\mathfrak{h}^{\perp}=\{S \in \mathfrak{g} \mid B(S, \mathfrak{h})=0\} .
$$

Under this identification, $\mathfrak{h}^{\perp}$ is the "conormal space" of $\mathfrak{h}$. Therefore, $T_{e K}^{*} X$ can be identified with $\mathfrak{p}$ and so for $g \in G, T_{g K}^{*} X=A d(g) \mathfrak{p}$. Let $\xi=g_{0} M N$ be a horocycle. Define

$$
\mathscr{N}^{0}(\xi)=\left\{(g K, A d(g) S) \mid g \in g_{0} M N, S \in \mathfrak{a}^{\prime}\right\}
$$

As $\mathfrak{a} \subset \mathfrak{p}, \mathscr{N}^{0}(\xi)$ can be viewed as a subset of $T^{*} X$. Lemma 3.2 shows $\mathscr{N}^{0}(\xi)$ is actually a subset of the conormal bundle of $\hat{\xi}$.

Theorem 3.1. Let $G$ be a semisimple Lie group of any real rank. Let $R_{\mu}$ be a Radon transform on horocycles with a weight function $\mu$ that is real-analytic and never zero. Let $f \in \mathscr{E}^{\prime}(X)$ and let $\xi=g_{0} M N$ be a given horocycle. Assume $R_{\mu} f=0$ on all horocycles in an open neighborhood of $\xi$. Then $\mathrm{WF}_{\mathrm{A}}(f) \cap$ $\mathscr{N}^{0}(\xi)=\varnothing$ where $\mathscr{N}^{0}(\xi)$ is given by $(3.1)$.

Radon transforms detect only microlocal singularities conormal to the set being integrated over (see, e.g., Lemma 3.2). This theorem implies that $R_{\mu} f$ does not detect all singularities of $f$ conormal to $\hat{\xi}$, but only those singularities corresponding to $S \in \mathfrak{a}^{\prime}$. If $G$ has rank one, then $\mathfrak{a}^{\prime}=\mathfrak{a} \backslash 0$ and so $\mathscr{N}^{0}(\xi)=$ $N^{*} \hat{\xi} \backslash 0$. In this case, all directions conormal to $\hat{\xi}$ are detected, and a strong support theorem is easier to prove [17]. A part of the proof of Theorem 3.1 is based on microlocal calculations in unpublished work of Guillemin [4], and the calculations that are not given below are in [17].

Proof of Theorem 3.1. As noted above, (2.1) allows the set $Z$ to be viewed as a subset of $X \times Y$. Let $N^{*} Z$ denote the conormal bundle of $Z$ in $T^{*}(X \times Y)$. The microlocal analysis of $R_{\mu}$ is discovered from the following diagram on cotangent spaces that corresponds to $(2.1)$ :

$$
\begin{aligned}
\Gamma= & N^{*} Z \backslash 0 \stackrel{\pi_{Y}}{\rightarrow} T^{*}(Y) \\
& \downarrow \pi_{X} \\
& T^{*}(X)
\end{aligned}
$$

where $\pi_{X}$ and $\pi_{Y}$ are the natural projections.

Without loss of generality, we can assume $\xi=\xi_{0}=M N$ is the identity coset in $Y$. The set $p_{Y}^{-1}\left(\xi_{0}\right)=M N / M \simeq N$ is the orbit of $M N$ in $Z=G / M$ (see [7, Theorem 3.4]). Let $\Gamma_{0}$ be the subset of $\Gamma$ lying above $p_{Y}^{-1}\left(\xi_{0}\right)$. The goal of the proof is to show $\pi_{Y}$ is an injective immersion on $\pi_{X}^{-1} \mathscr{N}^{0}\left(\xi_{0}\right) \subset \Gamma_{0}$. This is a kind of "local Bolker assumption" and, under this assumption, the calculus of analytic elliptic Fourier integral operators (see [20] for the $C^{\infty}$ category, [15, $18]$ for the real-analytic category) can be applied to prove $\mathrm{WF}_{\mathrm{A}}(f) \cap \mathscr{N}^{0}\left(\xi_{0}\right)=$ $\varnothing$. An analogous argument is given in [1]. 
By [17, Lemma 3.2], the map $\pi_{X}: \Gamma_{0} \rightarrow N^{*} \hat{\xi}_{0} \backslash 0$ is an injective immersion. So, let

$$
\bar{\rho}=\pi_{Y} \circ \pi_{X}^{-1}: N^{*} \hat{\xi}_{0} \backslash 0 \rightarrow T_{\xi_{0}}^{*} Y .
$$

To finish the proof, we will show that $\bar{\rho}$ is an injective immersion on domain $\mathscr{N}^{0}\left(\xi_{0}\right)$ (Lemma 3.3), and along the way, we will show $R_{\mu}$ is an elliptic Fourier integral operator associated to $\Gamma$. The following is Lemma 3.3 of [17].

Lemma 3.2. Let $G=K A N$ be the Iwasawa decomposition of a semisimple Lie group of any real rank and let $M$ be the centralizer of $A$ in $K$. Let $\xi_{0}$ be the horocycle $M N$. Then the conormal space $N^{*} \hat{\xi}_{0} \backslash 0=\{(g K, A d(g) S) \mid g \in$ $M N, S \in \mathfrak{a} \backslash 0\}$ and the cotangent space $T_{\xi_{0}}^{*} Y=\mathfrak{a}+\mathfrak{n}$. The map $\bar{\rho}=\pi_{Y} \circ \pi_{X}^{-1}$ is projection on the second factor:

$$
\begin{aligned}
\bar{\rho}: N^{*} \hat{\xi}_{0} \backslash 0 & \rightarrow T_{\xi_{0}}^{*} Y, \\
\bar{\rho}(g K, A d(g) S) & =A d(g) S .
\end{aligned}
$$

We can now prove that $R_{\mu}$ is an elliptic Fourier integral operator. The Schwartz kernel of $R_{\mu}$ is integration over the manifold $Z$ (e.g., [16]), so $R_{\mu}$ is a Fourier integral distribution associated to $\Gamma[13, \S 2.4]$. Because of $[17$, Lemma 3.2] (see above (3.3)), the projection $\pi_{X}$ maps to $T^{*} X \backslash 0$. Using (3.4) and the fact that $A d(g)$ is always injective, one sees that $\pi_{Y}$ maps to $T^{*} Y \backslash 0$. This implies $R_{\mu}$ is a Fourier integral operator associated with $\Gamma$ [20]. Since $\mu$ is nowhere zero, $R_{\mu}$ is elliptic. Finally, we will check:

Lemma 3.3. The map $\bar{\rho}$ in (3.3)-(3.4) is an injective immersion on domain $\mathscr{N}^{0}\left(\xi_{0}\right)$.

Proof. Using Lemma 3.2, we first prove $\bar{\rho}$ is injective. Let $g_{1}=m_{1} n_{1} \in M N$ and $S_{1} \in \mathfrak{a}^{\prime}$ be such that $A d\left(g_{1}\right) S_{1} \in \mathfrak{a}$. As $M$ centralizes $\mathfrak{a}, A d\left(n_{1}\right) S_{1} \in \mathfrak{a}$. We now prove $n_{1}=e$. Because $S_{1}$ is regular, the following map is an analytic diffeomorphism [11, Lemma 1.5 p. 403]:

$$
\begin{aligned}
\phi: N & \rightarrow \mathfrak{n}, \\
\phi(n) & =\operatorname{Ad}(n) S_{1}-S_{1} .
\end{aligned}
$$

As $\phi\left(n_{1}\right) \in \mathfrak{a} \cap \mathfrak{n}$ and $\mathfrak{a} \cap \mathfrak{n}=\{0\}$, we see that $\phi\left(n_{1}\right)=0$. Therefore, $n_{1}=e$ and so $g_{1}=m_{1} \in M$. This proves that $\bar{\rho}$ is injective.

To show $\bar{\rho}$ is an immersion at $\left(g_{1} K, A d\left(g_{1}\right) S_{1}\right) \in \mathscr{N}^{0}\left(\xi_{0}\right)$, we construct an arbitrary path through this point and calculate derivatives. Let $g(t)$ be a path in $M N$ with $g(0)=g_{1}$ and $\frac{d g}{d t}(0)=A d\left(g_{1}\right) V_{1}$ for some $V_{1} \in \mathfrak{m}+\mathfrak{n}$. Now, let $S(t)$ be a path in $\mathfrak{a}^{\prime}$ with $S(0)=S_{1}$ and $\frac{d S}{d t}(0)=T_{1} \in \mathfrak{a}$. Then $v(t)=(g(t) K, A d(g(t)) S(t))$ is a path in $\mathscr{N}^{0}\left(\xi_{0}\right)$ with derivative $\frac{d v}{d t}(0)=$ $\left(\operatorname{Ad}\left(g_{1}\right) V_{1}, A d\left(g_{1}\right)\left[a d\left(V_{1}\right) S_{1}+T_{1}\right]\right)$. Assume $\frac{d \bar{\rho} \circ v}{d t}(0)=A d\left(g_{1}\right)\left[a d\left(V_{1}\right) S_{1}+T_{1}\right]$ is zero. Therefore, $a d\left(V_{1}\right) S_{1}=-T_{1}$. As $\mathfrak{m}$ centralizes $\mathfrak{a}$, we can assume $V_{1} \in$ $\mathfrak{n}$. Since the derivative of the function in (3.5) maps to $\mathfrak{n}, \operatorname{ad}\left(V_{1}\right) S_{1} \in \mathfrak{n}$, so $T_{1} \in \mathfrak{n}$. Now, because $\mathfrak{a} \cap \mathfrak{n}=\{0\}, \operatorname{ad}\left(V_{1}\right) S_{1}=0$. Finally, since the derivative of the map (3.5) is injective, $V_{1}$ is zero. This proves that $\frac{d v}{d t}(0)=0$.

\section{THE SUPPORT THEOREM}

First we construct special codimension one hypersurfaces in $X=G / K$ that are made up of horocycles. A powerful theorem of Hörmander, Kawai, and 
Kashiwara gives information about the analytic wavefront set of a distribution at the boundary of its support. Next, we use this theorem, the special codimension one surfaces, and Lemma 4.3 to "eat away at" supp $f$ and show that supp $f$ is small when $f$ satisfies the hypotheses of Theorem 4.1 (below).

The hypersurfaces in $X$ are defined as follows. Let $T \subset \mathfrak{a}$ be a complete codimension one surface without boundary and let $g_{0} \in G$. Then the horocyclic surface $\mathscr{H}\left(g_{0}, T\right) \subset X$ is defined to be

$$
\mathscr{H}\left(g_{0}, T\right)=\bigcup_{H \in T}\left(g_{0} \exp (H) N\right) K
$$

$\mathscr{H}\left(g_{0}, T\right)$ is an imbedded complete codimension one analytic surface in $X$ because $T$ is codimension one in $\mathfrak{a}$ and by the Iwasawa Decomposition Theorem [11]: the map $a \times N \ni(H, n) \rightarrow \exp (H) n K$ is an analytic bijection onto $X$. Because the map $K / M \times A \rightarrow Y,(k M, a) \rightarrow k a M N$ is an analytic diffeomorphism [7] and $A$ normalizes $N$, it is sufficient to use $k_{0} \in K$ rather than $g_{0} \in G$ in (4.1). Also, because $M$ centralizes $N$, " $M N$ " has been replaced by " $N$ " in (4.1). If $P \subset \mathfrak{a}$ is an affine hyperplane, then $\mathscr{H}\left(k_{0}, P\right)$ will be called a horocyclic plane.

Theorem 4.1. Let $G$ be a semisimple Lie group of real rank greater than one. Let $R_{\mu}$ be a Radon transform on horocycles with a weight function $\mu$ that is real-analytic and never zero. Let $f \in \mathscr{E}^{\prime}(X)$ and let $\mathscr{A}$ be an open connected subset of the set of all horocyclic planes. Assume $R_{\mu} f(\xi)=0$ for all horocycles $\xi$ in all horocyclic planes in $\mathscr{A}$ and that some horocyclic plane in $\mathscr{A}$ is disjoint from $\operatorname{supp} f$. Then $\bigcup\{\mathscr{H} \mid \mathscr{H} \in \mathscr{A}\}$ is disjoint from supp $f$.

In $\S 5$, a stronger result than Theorem 4.1 is described in which nonplanar horocyclic surfaces are used. Helgason's support theorem [10] for (domain $\mathscr{E}^{\prime}(X)$ and) horocycles exterior to a geodesic ball, $B$, follows from Theorem 4.1 because $\mathscr{A}=\{\xi \mid \hat{\xi} \cap B=\varnothing\}$ is open and connected and because geodesic balls are determined by the horocyclic planes exterior to them. ${ }^{1}$

Using the techniques in the proof of Theorem 4.1, one can prove local support theorems such as the following.

Theorem 4.2. Let $G$ be a semisimple Lie group of real rank greater than one. Let $R_{\mu}$ be a Radon transform on horocycles with a weight function $\mu$ that is real-analytic and never zero. Let $\mathbb{S}$ be a geodesic sphere in $X$. Let $f \in \mathscr{E}^{\prime}(X)$ and assume supp $f$ is disjoint from $\mathbb{S}$. Assume $R_{\mu} f$ is zero on all horocycles meeting $\mathbb{S}$. Then $f=0$ inside of $\mathbb{S}$.

The hypotheses of Theorem 4.2 allow supp $f$ to include points outside $\mathbb{S}$. The choice of $S$ is somewhat arbitrary and local support theorems can be proved for other sets as in [2, 17].

Proof of Theorem 4.1. To prove the theorem we "eat away" at supp $f$. Because $\mathscr{A}$ is connected and some horocyclic plane in $\mathscr{A}$ is disjoint from supp $f$, there is a horocyclic plane $\mathscr{H}_{0}=\mathscr{H}\left(k_{0}, P_{0}\right)$ that meets supp $f$ and such that

\footnotetext{
${ }^{1}$ First assume the ball is centered at the origin in $X ; x$ is a point in its boundary; and that $x=k_{0} a_{0} K$ for some $k_{0} \in K$ and $a_{0} \in A$. Now [8, Lemma 5.4] can be used to choose a plane $P \in \mathfrak{a}$ through $\log \left(a_{0}\right)$ such that $B$ is on one side of $\mathscr{H}\left(k_{0} a_{0}, P\right)$.
} 
$f$ is zero on one side of $\mathscr{L}_{0}$ (one argument is to construct a path in $\mathscr{A}$ from a plane outside supp $f$ to one meeting supp $f$ and then let $\mathscr{H}_{0}$ be the first horocyclic plane in that path that meets supp $f$ ).

The goal is to get a contradiction. Let $x_{0}=g K \in \operatorname{supp} f \cap \mathscr{H}_{0}$ where $g=k_{0} \exp \left(H_{0}\right) n_{0}$ for some $H_{0} \in P_{0}$ and $n_{0} \in N$, and let $\hat{\xi}_{0}$ be the horocycle in $\mathscr{H}_{0}$ containing $x_{0}$. From now on, we will let $P_{0}^{\perp}$ denote the set of vectors in a normal to $P_{0}$ under the Killing form. Now, let $S_{0} \in P_{0}^{\perp} \backslash 0$. The next lemma implies that $\left(x_{0}, \operatorname{Ad}(g) S_{0}\right) \in N^{*} \mathscr{L}_{0}$.

Lemma 4.3. Let $P \subset \mathfrak{a}$ be an affine hyperplane and let $k_{0} \in K$. Then the conormal bundle of $\mathscr{H}\left(k_{0}, P\right)$ is

$$
N^{*} \mathscr{H}\left(k_{0}, P\right)=\left\{(g K, A d(g) S) \mid g \in k_{0} \exp (P) N, S \in P^{\perp}\right\} .
$$

Proof. Let $g=k_{0} \exp \left(H_{0}\right) n_{0}$ for some $H_{0} \in P$ and $n_{0} \in N$. Now $\hat{\xi}=$ $\left(k_{0} \exp \left(H_{0}\right) N\right) K$ is the horocycle in $\mathscr{H}\left(k_{0}, P\right)$ containing $g K$. To show the right-hand set in (4.2) is contained in $N^{*} \mathscr{H}\left(k_{0}, P\right)$, we will show the covector $(g K, A d(g) S)$, for $S \in P^{\perp}$, is conormal to $\hat{\xi}$ as well as to the tangent vectors in $T \mathscr{H}\left(k_{0}, P\right)$ that are transversal to $\hat{\xi}$. By Lemma 3.2, $(g K, A d(g) S)$ is conormal to $\hat{\xi}$. Now, let $H:(-1,1) \rightarrow P$ be any differentiable path with $H(0)=H_{0}$. Clearly $A d(g) S$ is conormal to the infinitesimal generator of the path $k_{0} \exp (H(t)) n_{0} K$ and so $(g K, A d(g) S) \in N^{*} \mathscr{H}\left(k_{0}, P\right)$. Now a dimension count shows that equality holds in (4.2). is:

However, a special case of a theorem of Kawai, Kashiwara, and Hörmander

Lemma 4.4 [14, Theorem 8.5.6]. Let $f \in \mathscr{E}^{\prime}(X)$ and assume $f$ is zero on one side of the horocyclic plane $\mathscr{H}_{0}$. Let $x_{0} \in \mathscr{H}_{0} \cap \operatorname{supp} f$ and let $\left(x_{0}, \omega\right) \in$ $N^{*} \mathscr{L}_{0} \backslash 0$. Then $\left(x_{0}, \omega\right) \in \mathrm{WF}_{\mathrm{A}} f$.

Hörmander's result is stated for arbitrary smooth hypersurfaces in $\mathbb{R}^{\mathrm{n}}$, but it can be proved in $X$ using real-analytic local coordinates and the fact that horocyclic planes are complete codimension one surfaces.

First assume $S_{0} \in \mathfrak{a}^{\prime}$. As $R_{\mu} f=0$ for horocycles near $\xi_{0}$, the conclusion of Theorem 3.1 implies $\left(x_{0}, A d(g) S_{0}\right) \notin \mathrm{WF}_{\mathrm{A}} f$. Using Lemmas 4.3 and 4.4, one sees that $\left(x_{0}, A d(g) S_{0}\right) \in \mathrm{WF}_{\mathrm{A}} f$. This contradiction proves the theorem if $S_{0} \in \mathfrak{a}^{\prime}$.

Now assume $S_{0} \notin \mathfrak{a}^{\prime}$ and $x_{0}$ is as above. Because $f$ has compact support, there is a $k_{1} \in K$ so near to $k_{0}$ and a direction $S_{1} \in \mathfrak{a}^{\prime}$ so near to $S_{0}$ and hyperplanes $P_{\text {out }}$ and $P_{\text {in }}$ in a both conormal to $S_{1}$ such that

(4.3) $x_{0} \in \mathscr{H}\left(k_{1}, P_{\text {in }}\right)$,

(4.4) for each parallel hyperplane $P$ between $P_{\text {out }}$ and $P_{\text {in }}$, the horocyclic planes $\mathscr{H}\left(k_{1}, P\right) \in \mathscr{A}$, and

(4.5) $\mathscr{H}\left(k_{1}, P_{\text {out }}\right) \cap \operatorname{supp} f=\varnothing$.

The horocyclic planes in (4.4) can be used to eat away at supp $f$ near $x_{0}$ as in the proof above because $S_{1} \in \mathfrak{a}^{\prime}$.

Proof of Theorem 4.2. The proof is done by contradiction. Let $B$ be the closed disk with boundary $\mathbb{S}$. Let $B_{1}=B \cap$ supp $f$; then $B_{1} \neq \varnothing$ and, under the hypotheses of Theorem 4.2, $B_{1} \cap \mathbb{S}=\varnothing$. Therefore, there is a horocyclic plane 
$\mathscr{H}_{0}=\mathscr{H}\left(k_{0}, P_{0}\right)$ (with $\left.\left(P_{0}^{\perp} \backslash 0\right) \subset \mathfrak{a}^{\prime}\right)$ that meets $B_{1}$ and such that $B_{1}$ is to one side of $\mathscr{H}_{0}$. Since $\mathscr{H}_{0}$ meets int $B$, Theorem 3.1 and Lemmas 4.3 and 4.4 can be used to show $f$ is zero near $\mathscr{K}_{0}$. This contradicts the choice of $\mathscr{H}_{0}$. Note that Lemma 4.3 can be applied because its hypotheses are satisfied locally in $B$ and its conclusion is local (see statement of Theorem 8.5.6 in [14]).

\section{Generalizations of Theorem 4.1}

Horocyclic planes are used in the statement of Theorem 4.1 because of their simple geometric nature-all conormals to the horocyclic plane $\mathscr{H}\left(k_{0}, P\right)$ come from the same subspace, $P^{\perp} \subset$ a (Lemma 4.3). However, a stronger theorem can be proven by using other hypersurfaces in $\mathfrak{a}$ to define the horocyclic surfaces (4.1). We will describe, in general, such a class of surfaces and then outline a specific example.

Let $T_{0}$ be a smooth complete hypersurface without boundary in a . Assume

$$
\forall H \in T_{0}, N_{H}^{*} T_{0} \backslash 0 \subset \mathfrak{a}^{\prime} .
$$

Here, $N_{H}^{*} T_{0}$ is the set of all vectors in a normal to $T_{0}$ at $H$ under the Killing form. Now let $\mathscr{T}$ be the collection of all translates in $\mathfrak{a}$ of $T_{0}$. Then the generalization of Theorem 4.1 holds for horocyclic surfaces $\mathscr{H}\left(k_{0}, T\right)$ for $k_{0} \in$ $K$ and $T \in \mathscr{T}$. Condition (5.1) is simply the requirement that all nonzero conormals to $T$ are regular (in $\mathfrak{a}^{\prime}$ ).

To prove this generalization of Theorem 4.1 , one must replace Lemma 4.3 by

$$
\begin{aligned}
N^{*} \mathscr{H}\left(k_{0}, T\right)=\{( & g K, A d(g) S) \mid g=k_{0} \exp (H) n, \\
& \left.\quad \text { for some } H \in T, n \in N, S \in N_{H}^{*} T\right\}
\end{aligned}
$$

(the proof is similar to the proof of Lemma 4.3). The proof of the stronger theorem is now essentially the same as for horocyclic planes with $S_{0} \in \mathfrak{a}^{\prime}$ because the wavefront set of $f$ conormal to $\mathscr{H}\left(k_{0}, T\right)$ is detected by data $R_{\mu} f$.

An example will be described in rank 2 . In this case, the walls of the Weyl chambers, $\mathfrak{a} \backslash \mathfrak{a}^{\prime}$, are rays with base at the origin. Choose two adjacent rays, $\mathbf{r}_{1}$ and $\mathbf{r}_{2}$. For $j=1,2$ let $\mathbf{H}_{j}$ be the open half-plane disjoint from $\mathbf{r}_{j}$ and with boundary perpendicular to $\mathbf{r}_{j}$ at the origin. Let $W=\mathbf{H}_{1} \cap \mathbf{H}_{2}$; then $W$ is a convex wedge. Choose a hyperbola with asymptotes $\partial \mathbf{H}_{1} \cup \partial \mathbf{H}_{2}$ and with one half in $W$. Let $T_{0}$ be that half-hyperbola in $W$. Then the nonzero conormals to $T_{0}$ lie in the open cone between $\mathbf{r}_{1}$ and $\mathbf{r}_{2}$ and so are in $\mathfrak{a}^{\prime}$. Therefore, $T_{0}$ satisfies (5.1). Therefore, the horocyclic surfaces defined by translates of $T_{0}$ satisfy the generalization of Theorem 4.1. In fact, one can make the hyperbola $T_{0}$ fit closer and closer to $\partial W$ and then show that the generalization of Theorem 4.1 is valid for horocyclic surfaces defined by translates of $\partial W$. These surfaces allow one to get inside the "convex hull" of supp $f$ because they are "convex". The analogous constructions of $T_{0}$ and the limiting set $\partial W$ are valid for spaces with rank greater than two.

\section{ACKNOWLEDGMENT}

The authors thank Victor Guillemin and Sigurdur Helgason for much inspiration over the years. They are also indebted to the referee for valuable comments on the form and content of the article. 


\section{REFERENCES}

1. J. Boman and E. T Quinto, Support theorems for real analytic Radon transforms on line complexes in $\mathbb{R}^{3}$, Trans. Amer. Math. Soc. 335 (1993), 877-890.

2. J. Globevnik, A support theorem for the X-ray transform, J. Math. Anal. Appl. 165 (1992), 284-287.

3. A. Greenleaf and G. Uhlmann, Non-local inversion formulas in integral geometry, Duke J. Math. 58 (1989), 205-240.

4. V. Guillemin, Some remarks on integral geometry, unpublished, 1975.

5. __ On some results of Gelfand in integral geometry, Proc. Sympos. Pure Math., vol. 43, Amer. Math. Soc., Providence, RI, 1985, pp. 149-155.

6. V. Guillemin and S. Sternberg, Geometric asymptotics, Amer. Math. Soc., Providence, RI, 1977.

7. S. Helgason, Duality and Radon transform for symmetric spaces, Amer J. Math. 85 (1963), 667-692.

8. __ An analog of the Paley-Wiener theorem for the Fourier transform on certain symmetric spaces, Math. Ann. 165 (1966), 297-308.

9. __ A duality for symmetric spaces, with applications to group representations, Adv. Math. 5 (1970), 153-180.

10. (2) 98 (1973), 451-479.

11. _ Differential geometry, Lie groups, and symmetric spaces, Academic Press, New York, 1978.

12. Groups and geometric analysis, Academic Press, New York, 1984.

13. L. Hörmander, Fourier integral operators. I, Acta Math. 127 (1971), 79-183.

14. _ The analysis of linear partial differential operators. I, Springer, New York, 1983.

15. A. Kaneko, Introduction to hyperfunctions, Kluwer, New York, 1989.

16. E. T. Quinto, The dependence of the generalized Radon transform on defining measures, Trans. Amer. Math. Soc. 257 (1980), 331-346.

17. 117 (1993), 179-186.

18. M. Sato, T. Kawai, and M. Kashiwara, Hyperfunctions and pseudodifferential equations, Lecture Notes in Math., vol. 287, Springer-Verlag, New York, 1973, pp. 265-529.

19. C. D. Sogge and E. M. Stein, Averages over hypersurfaces smoothness of generalized Radon transforms, J. Analyse Math. 54 (1990), 165-188.

20. F. Treves, Introduction to pseudodifferential and Fourier integral operators. I, Plenum Press, New York, 1980.

21. P. D. Lax and R. S. Phillips, A local Paley-Wiener Theorem for the Radon transform of $L^{2}$ functions in a non-euclidean setting, Comm. Pure Appl. Math. 35 (1982), 531-554.

Department of Mathematics, Tufts University, Medford, Massachusetts 02155

E-mail address: gonzalez@jade tufts.edu

$E$-mail address: equint o@math.tufts.edu 